\title{
Methodik und Ergebnisse von Untersuchungen des Erythrozyten- und Hämoglobingehaltes frischgefangener Seefische
}

\author{
Aus dem Institut für Meereskunde der Universität Kiel \\ und der Biologischen Anstalt Helgoland \\ Von Heinz Schaefer \\ (Mit 3 Abbildungen und 2 Tabellen im Text)
}

Unsere Kenntnisse vom Blut der Seefische sind noch sehr gering. Hierfür dürften zwei Gründe verantwortlich sein: Erstens bietet das Fischblut der Untersuchung große Schwierigkeit wegen der sehr schnellen Gerinnung und der sehr geringen Menge - größenordnungsmäßig entspricht die Blutmenge bei den Fischen etwa 1/50 des Körpergewichts -, zweitens ist das Versuchsmaterial, insbesondere das aus der offenen See stammende, schwer zugänglich.

Bei der Bestimmung der Erythrozytenzahlen und des Hämoglobingehaltes begnügte sich daher bisher die Mehrzahl der Autoren damit, in Küstennähe lebende Fische in ein Landaquarium bringen zu lassen und dort nach einer gewissen Adaptationszeit die Analysen durchzuführen. Es ist indes fraglich, ob die so erhaltenen Werte mit denen von frisch aus ihrem natürlichen Lebensraum gefangenen Fischen übereinstimmen.

Die Hämoglobinbestimmung wurde meist analog der klinischen SahliMethode durchgeführt, die nach DruCkREy et al. (1952) aber große Fehlermöglichkeiten einschließt. Häufig bestimmte man den Blutfarbstoffgehalt auch indirekt, indem man aus der Bluteisenkonzentration auf die Hämoglobinmenge schloß. Hall und Gray (1929) vermeiden aber ausdrücklich, den Gehalt an Eisen in Hämoglobin umzurechnen. Wir wissen nicht, wieviel Eisen, das nicht an Hämoglobin gebunden ist, im Blut der verschiedenen Fischarten vorliegt.

In vielen Fällen wurde bisher nur der Hämoglobingehalt festgestellt, ohne die Zahl und die Größe der Erythrozyten zu erfassen, oder es wurden nur Angaben über die Größe der roten Blutkörperchen gemacht.

*) Deutscher Beitrag zum Polarfrontprogramm des Internationalen Geophysikalischen Jahres 1958.

*) Der Deutsdien Forschungsgemeinschaft danke ich ergebenst für die Gewährung eines Stipendiums und einer Sachbeihilfe. 
Die häufig fragmentarischen Angaben und das Fehlen von Daten von vielen wichtigen Fischarten aus der Nordsee und dem Nordatlantik machten eine weitere Untersuchung des Fischblutes notwendig. Dabei schien es von großer Bedeutung zu sein, das Blut von frischgefangenen Fischen zu analysieren, um eine klare Vorstellung davon zu bekommen, wie sich das Blut direkt nach dem Fang der Tiere aus dem natürlichen Lebensraum zusammensetzt. Für vergleichende Betrachtungen sollten die Untersuchungen auf eine große Artenzahl ausgedehnt und vornehmlich solche Arten studiert werden, von denen wir bisher keine oder nur ungenügende hämatologische Kenntnisse haben. Dabei sollten auch die Beziehungen zwischen Zahl und Oberfläche der roten Blutkörperchen und Hämoglobingehalt überprüft werden.

Von Interesse war ferner die Frage, ob Unterschiede im Blutbild von Fischen aus verschiedenen geographischen Gebieten festzustellen sind, und inwieweit sich die Lebensweise der Tiere in der Zahl und der Größe der Erythrozyten und im Hämoglobingehalt widerspiegelt.

Eine solche Untersuchung war nur an Bord eines Schiffes möglich. Es galt daher, möglichst einfache und dennoch genaue Methoden zu finden, die die Durchführung des Vorhabens auf See gestatteten.

Über die ausgearbeitete Methode und über die gewonnenen Ergebnisse wird im folgenden berichtet. Die Untersuchungen erfolgten an Bord des deutschen Fischereiforschungsschiffes "Anton Dohrn" anläßlich der beiden Forschungsfahrten im Internationalen Geophysikalischen Jahr. Dadurch war es möglich, Fische aus der Nordsee bis zu den isländisch-grönländischen Meeren zu bearbeiten. Auf einer weiteren Ausfahrt im Juni wurden zusätzlich mehrere Fischarten in der Nordsee untersucht. Hierbei konnten jedoch keine Hämoglobinbestimmungen erfolgen. Von den meisten Fangstationen liegen gleichzeitig gemachte hydrographische Analysen vor, so daß die Wasserkörper am Fangort genau durch Salzgehalt, Temperatur und Sauerstoffmenge charakterisiert werden können.

\section{Methodik}

Die Fische wurden sofort nach dem Fang durch Schlag getötet. Nach Säuberung und Trocknung des Operationsfeldes öffnete ich die Caudalgefäße hinter dem After durch einen kurzen Einstich mit dem Skalpell, verwarf die ersten Blutstropfen und fing das hervorquellende Blut in Paraffinschälchen auf, die einige Körnchen Vetren (Heparin) enthielten. Sofort anschließend erfolgten die Verdünnung des Blutes mit Hayemscher Lösung in der bekannten Verdünnungspipette, die Überführung von $0,038 \mathrm{ml}$ Blut in Transformationsflüssigkeit $(0,2 \mathrm{~g}$ Kaliumferricyanid und $0,2 \mathrm{~g}$ Kaliumcyanid ad $1000 \mathrm{ml}$ dest. Wasser) und die Beschickung der Bürkerschen Zählkammern nach Vorschrift. Wenn es die Schiffsbewegungen gestatteten oder nur ein bis zwei Fische zu bearbeiten waren, konnten die roten Blutkörperchen sofort gezählt und vermessen werden. War dies nicht möglich, so wurden beide Liniennetze der Zählkammern bei Verwendung von Okular $12 \times$ und Objektiv $10 \times$ so in das Blickfeld des Mikroskops gebracht, daß eine möglichst große Zahl von kleinsten Quadraten sichtbar war, ein Grünfilter eingeschoben und unter Verwendung eines Elektronenblitzes photographiert.

Das übliche Verfahren der Hämoglobinbestimmung durch Überführen in salzsaures Hämatin mußte wegen Ungenauigkeit verworfen werden. Störend 
ist hierbei auch das Einhalten einer exakten Ablesefrist, was an Bord oft nicht möglich ist. Statt dessen fand die Methode nach Betke und Savelsberg (1949/ 50) Anwendung. Vor jedem Fang stellte ich eine genügende Anzahl von Penicillinfläschchen mit Transformationsflüssigkeit bereit, in welche nach dem Fang nur noch das Blut einpipettiert zu werden brauchte. Nach Durchmischung konnten die Fläschchen abgestellt werden, da der sich bildende Komplex von Cyanhämiglobin mindestens zwei Tage lang stabil ist. Nach Abwarten einer ruhigen Schiffslage erfolgte die Ablesung der Extinktion im ELCo II (Zeiss) ${ }^{3 * * * *}$ ). Diese Methode ist sehr genau und-für Bordzwecke sehr geeignet. Eichungen bestätigten die Anwendbarkeit der Methode.

Mit dem angegebenen photographischen Verfahren können gleichzeitig etwa vier Fische bearbeitet werden. Die Tiere werden nebeneinander auf den Laboratoriumstisch gelegt, dann werden die Blutgefäße angeschnitten, das Blut gesammelt, und so werden in der Folge die einzelnen Arbeitsgänge jeweils hintereinander durchgeführt. Vor dem Pipettieren müssen die Blutproben leicht durchgeschüttelt werden, da die Sedimentationszeit bei manchen Arten sehr gering ist. Bei einiger Übung benötigt die Untersuchung nur wenige Minuten. Die Auswertung der Photogramme kann an Land erfolgen.

Als blutgerinnungshemmendes Mittel hat sich Heparin in substantia (Vetren) in Verbindung mit paraffinierten Auffangschälchen bestens bewährt, während Oxalat in Vorversuchen häufig versagte. Desgleichen wurde die Hayemsche Lösung zur Blutverdünnung für die Zählung der Erythrozyten mit Erfolg angewendet. In keinem Fall trat eine Formveränderung der Korpuskeln auf.

$$
\text { Ergebnisse }
$$

Es wurden folgende Arten untersucht:

Ordnung Gadiformes

Kabeljau, Dorsch Schellfisch

Köhler

Wittling

Blauer Wittling

Seehecht

Brosme, Lumb
Gadus morrhua L., Syn. Gadus callarias L.

Melanogrammus aeglefinus L., Syn.: Gadus aeglefinus $\mathrm{L}$.

Pollachius virens L., Syn.: Gadus virens L.

Merlangius merlangus L., Syn.: Gadus merlangus L.

Micromesistius poutassou Risso, Syn.: Gadus pouiassou Risso

Merluccius merluccius L., Syn.: Merluccius vulgaris Flem.

Brosme brosme Müller, Syn.: Brosmius brosme Ascanius

Ordnung Marcruriformes

Grenadierfisch

Macrourus rupestris Gunner

***) Das ELCO II wurde Herrn Prof. Dr. Bückmann, Biologische Anstalt Helgoland, von der Deutschen Forschungsgemeinschaft zur Verfügung gestellt. 
Bd. VIII, H. 1: Schaefer. Erythrozyten- und Hämoglobingehalt frischgefarigener Seefische 71

Ordnung Perciformes

Unterordnung Percoidei

Bastardmakrele Trachurus trachurus L., Syn.: Caranx trachurus L.

Petermännchen Trachinus draco L.

Streifenbrasse Spondyliosoma cantharus L., Syn.: Cantharus lineatus Mont.

Unterordnung Blennioidei

Katfisch, Steinbeißer Anarhichas lupus L.

gefleckter Katfisch Anarhichas minor Olafsen

Wolfsfisch Lycodes esmarki Collett.

Unterordnung Scombroidei

Makrele Scomber scombrus L.

Unterordnung Cottoidei

Rotbarsch

Sebastes marinus L.

Rot-, Tiefen-,

Schnabelbarsch

Sebastes mentella Travin

Knurrhahn

Trigla cuculus L., Syn.: Trigla pini BI

Grauer Knurrhahn

Trigla gurnardus $\mathrm{L}$.

Seehase, Lump

Cyclopterus lumpus L.

Unterordnung Callionymoidei

Leyerfisch Callionymus lyra $\mathrm{L}$.

Ordnung Clupeiformes

Hering Clupea harengus $\mathrm{L}$.

Finte Alosa fallax Lac.

Ordnung Lophiiformes

Seeteufel, Angler Lophius piscatorius L.

Ordnung Heterosomata

Familie Pleuronectidae

schwarzer Heilbutt Reinhardtius hippoglossoides Walbaum

Doggerscharbe Hippoglossoides platessoides Fabricius, Syn.: Drepanopsetta platessoides Walbaum

Limande

Microstomus kitt Walbaum, Syn.: Pleuronectes microcephalus Donov.

Kliesche

Limanda limanda L., Syn.: Pleuronectes limanda

Scholle

L.

Flunder

Pleuronectes platessa $\mathrm{L}$.

Familie Bothidae

Scheefsnut

Platichthys flesus L., Syn.: Pleuronectes flesus L.

Lepidorhombus whiff-iagonis Walbaum, Syn.: Lepidorhombus whiff Walbaum 
Bei der systematischen Einordnung der Plattfische folgte ich Norman (1934), bei der der übrigen Fische Berg (1958).

Von den genannten Arten sind meines Wissens nur für 9 Arten vollständige Angaben über Erythrozytenzahl und -größe und Hämoglobingehalt gemacht worden, von weiteren 9 Arten liegen in der Literatur nur Angaben über die Erythrozythengröße vor. Daten betreffend Fangtiefe und Temperatur sind aus der Tabelle 1 zu entnehmen. Der Salzgehalt der Wasserkörper ist wegen der nur sehr geringen Schwankungen nicht aufgeführt. Er betrug im Durchschnitt 35\%. Die Sättigung des Wassers mit Sauerstoff belief sich in den Fangtiefen durchweg auf über $85 \%$.

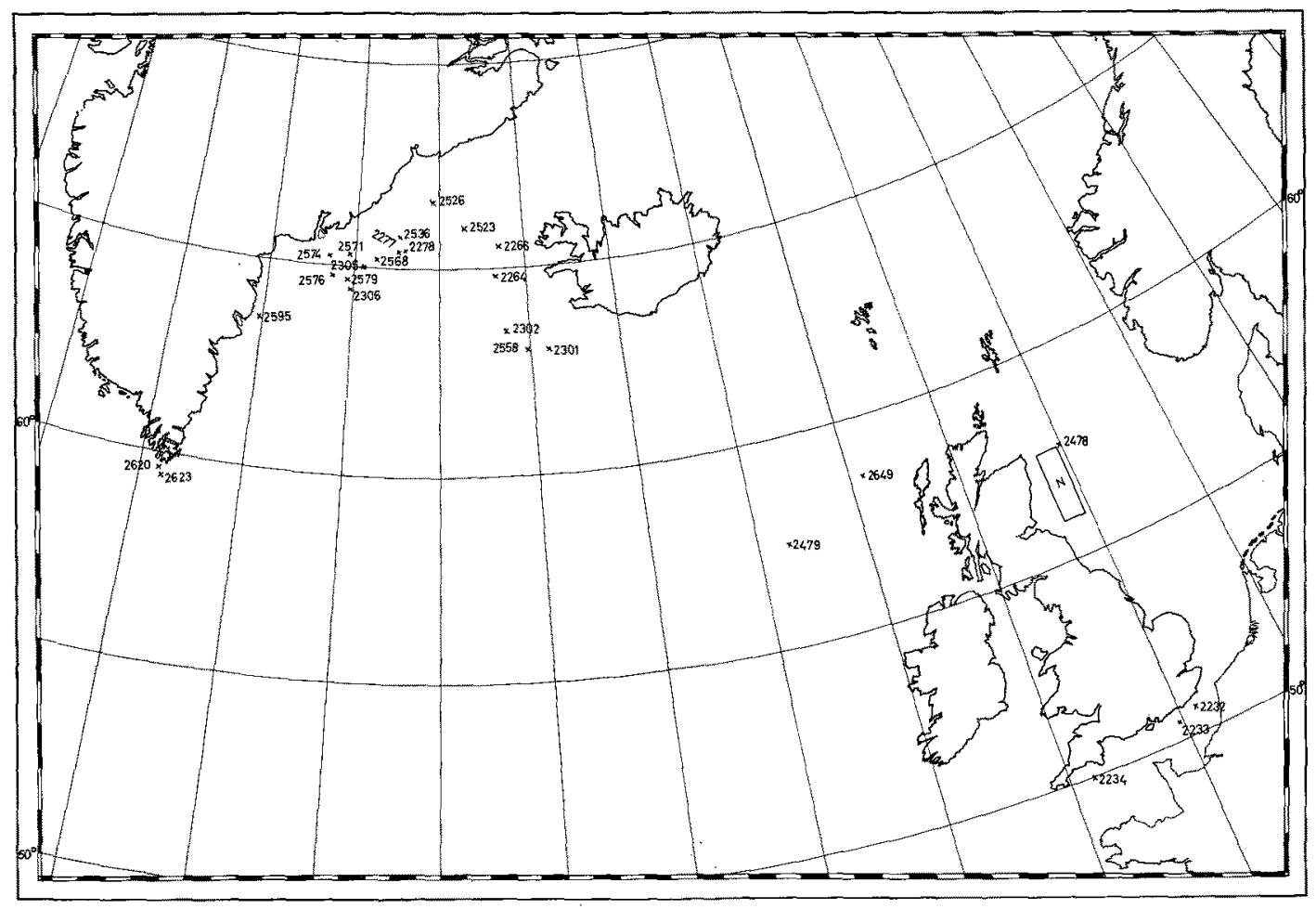

Abb. 1: Karte der Fangstationen

Die Ergebnisse sind in Tabelle 1 niedergelegt. Die Arten sind nach fal-lender Erythrozytenzahl geordnet. Die Fangplätze mit Stationsnummern sind aus Abbildung 1 ersichtlich. Wurden Exemplare einer Art an verschiedenen Stationen im Bereich Island-Grönland erfaßt, dann werden nur Gesamtdurchschnittswerte und gegebenenfalls die Streuung (Standardabweichung) mit Angabe der Stationen aufgeführt. Wurden Fische einer Art zu verschiedenen Jahreszeiten bzw. in gemäßigten und in kälteren Meeren gefangen, dann werden die Werte gesondert, aber in Zusammenhang mit der Art, angegeben (vergl. Kabeljau, Wittling). Die Befunde bei Männchen und Weibchen sind zum Teil verschieden. Da aber eine Tendenz nicht erkennbar ist, werden die Durchschnittswerte ohne Rücksicht auf das Geschlecht aufgeführt. 

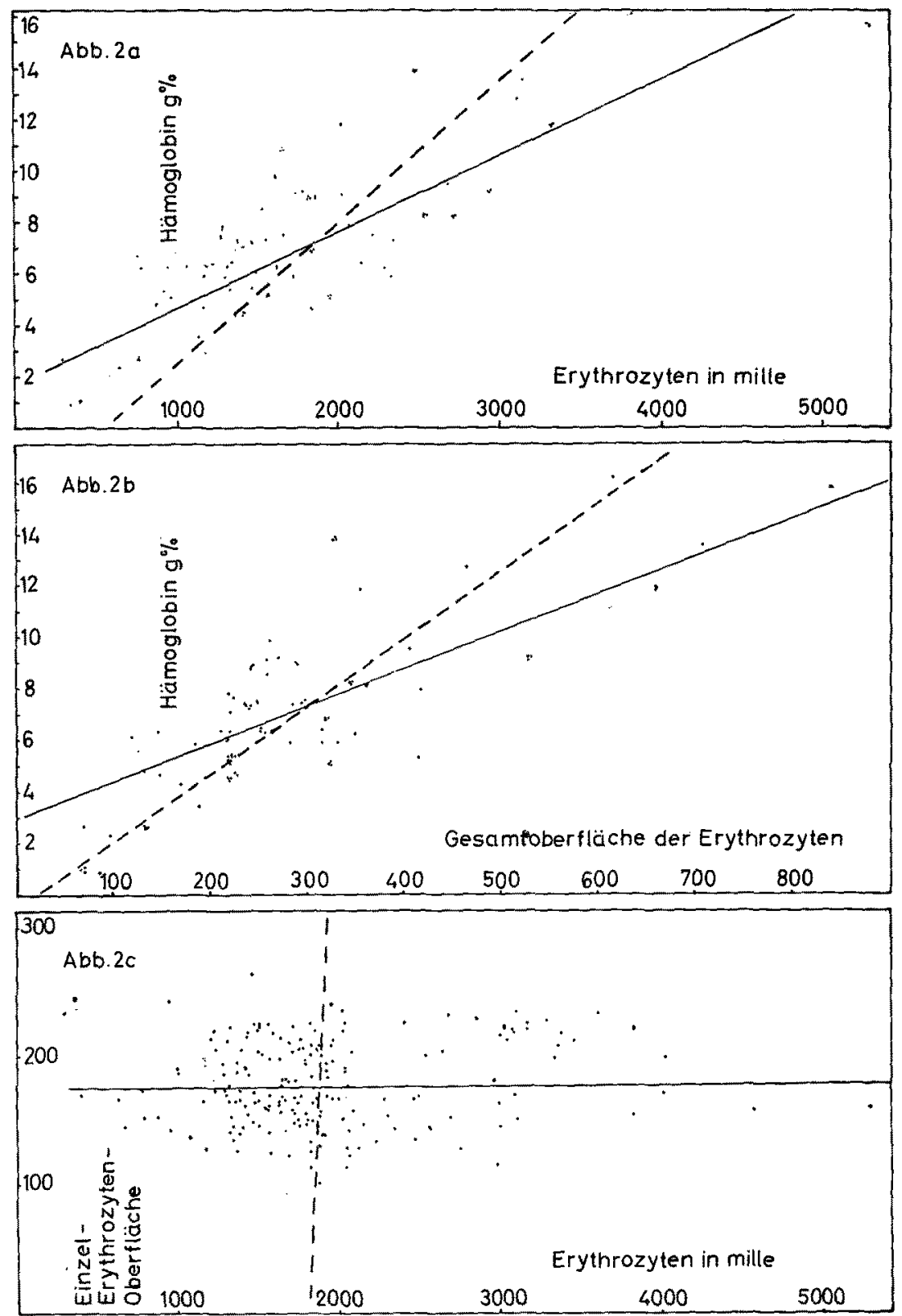

Abb. 2a: Beziehung Erythrozytenzahl zu Hämoglobingehalt

Abb. 2b: Beziehung Gesamtoberfläche der Erythrozyten zu Hämoglobingehalt Abb. 2c: Beziehung Erythrozytenzahl zu Oberfläche der einzelnen Erythrozyten

Die erhaltenen Daten schwanken sehr stark von Art zu Art. Auch innerhalb der Species werden beträchtliche Streuungen beobachtet. Es sind, wie schon von mehreren Autoren beschrieben wurde, drei Gruppen von Fischen erkennbar (vergl. auch Abbildung 3): solche mit stark konzentriertem Blut, d. h. großer Erythrozytenzahl und hohem Hämoglobingehalt (Makrele, Hering u. a.), solche mit sehr verdünntem Blut (Lophius) und dazwischen die große Gruppe der Fische mit mittleren Werten. 
Tabelle 1

\begin{tabular}{|c|c|c|c|c|c|}
\hline 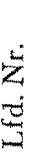 & 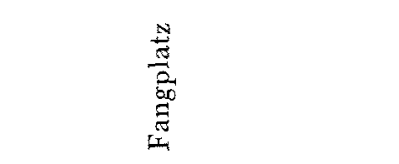 & 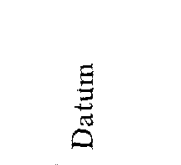 & 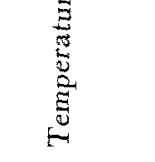 & 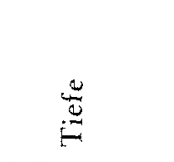 & $\Sigma$ \\
\hline 1 & Nordsee $(\mathrm{N}, 2478)$ & $2.6 .-7.8$ & $9-11,3^{0}$ & $80-95 \mathrm{~m}$ & Makrele \\
\hline 2 & Engl. Kanal (2232) & 1.3 & $6,9^{0}$ & $50 \mathrm{~m}$ & Leyerfisch \\
\hline 3 & Engl. Kanal (2232) & 1.3. & $6,9^{0}$ & $50 \mathrm{~m}$ & Finte \\
\hline 4 & Nordsee (N) & $2.6 .-7.8$ & $9-10,2^{0}$ & $65-100 \mathrm{~m}$ & Hering \\
\hline 5 & Engl. Kanal (2234) & 2.3 & $9,6^{0}$ & $72 \mathrm{~m}$ & Streifenbrasse \\
\hline 6 & westl. Schottland $(2479,2649)$ & $9.8 .-23.9$ & $9-10^{0}$ & $156-200 \mathrm{~m}$ & Scheef snut \\
\hline 7 & Engl. Kanal (2233) & 1.3 & $8,5^{0}$ & $53 \mathrm{~m}$ & Knurrhahn \\
\hline 8 & Engl. Kanal (2232) & 1.3 & $6,9^{\circ}$ & $50 \mathrm{~m}$ & Petermännchen \\
\hline 9 & westlich Schottland (2479) & 9.8. & $9,0^{0}$ & $156 \mathrm{~m}$ & grauer Knurrhahn \\
\hline 10 & Engl. Kanal (2234) & 2.3 & $9,6^{0}$ & $72 \mathrm{~m}$ & Bastardmakrele \\
\hline 11 & Island-Grönland (2526) & 22.8 & $0,40^{\circ}$ & $400 \mathrm{~m}$ & schwarzer Heilbutt \\
\hline 12 & Nordseee $(\mathrm{N})$ & 1.6.-6.8. & $8.7-9.8^{\circ}$ & $60-92 \mathrm{~m}$ & Kabeljau \\
\hline & Grönland (2574) & 5.9. & $6,6^{0}$ & $195 \mathrm{~m}$ & Kabeljau \\
\hline & Grönland (2277) & 19.3 & $6,9^{\circ}$ & $310 \mathrm{~m}$ & Kabeljau \\
\hline 13 & Nordsee $(N)$ & $4.6 .-12.6$ & $8-10,2^{0}$ & $70-115 \mathrm{~m}$ & Kliesche \\
\hline 14 & Island (2558) & 29.8 & $7,3^{0}$ & $450 \mathrm{~m}$ & Brosme \\
\hline 15 & Nordsee $(\mathrm{N})$ & 2. $6 .-14.6$ & $8-10,4^{0}$ & $47-104 \mathrm{~m}$ & Schellfisch \\
\hline 16 & westlid Schottland (2649) & 23.9 & $\mathrm{ca} .10^{\circ}$ & $207 \mathrm{~m}$ & Seehedht \\
\hline 17 & Nordsee $(\mathrm{N})$ & 1. $6-13.6$ & $9-10^{0}$ & $55-94 \mathrm{~m}$ & Scholle \\
\hline 18 & westlich Schottland (2649) & 23.9 & ca. $10^{\circ}$ & $207 \mathrm{~m}$ & Köhler \\
\hline \multirow[t]{4}{*}{19} & Island $(2264)$ & $16.3 .-$ & $4,4^{0}$ & $220 \mathrm{~m}$ & Rotbarsch \\
\hline & Grönland (2306) & 4.4 & $4,9^{0}$ & $405 \mathrm{~m}$ & Rotbarsch \\
\hline & Grönland (2623, & $21.8 .-$ & $0,1-$ & $195-$ & \\
\hline & $\begin{array}{l}2571,2574 \\
2595)\end{array}$ & 9.9 & $4,9^{0}$ & $380 \mathrm{~m}$ & $\begin{array}{l}\text { Rotbarsch } \\
\text { gesamt }\end{array}$ \\
\hline 20 & Grönland $(2568,2576)$ & $4.9 .-5.9$ & ca. $6,5^{\circ}$ & $450-480 \mathrm{~m}$ & Blauer Wittling \\
\hline 21 & Nordsee $(\mathrm{N})$ & 3. 6. & $9,0^{0}$ & $78 \mathrm{~m}$ & Flunder \\
\hline 22 & Nordsee (N) & $4.6 .-12.6$ & $9,6-10,2^{0}$ & $75=-115 \mathrm{~m}$ & Wittling \\
\hline & Engl. Kanal (2232) & 1. 3 & $6,9^{0}$ & $55 \mathrm{~m}$ & $\begin{array}{l}\text { Wittling } \\
\text { gesamt }\end{array}$ \\
\hline 23 & Grönland (2620) & 15.9 & $3-4^{0}$ & $130 \mathrm{~m}$ & Doggerscharbe \\
\hline 24 & Nordsee (N) & 4. $6 .-14.6$ & $8,5-10,4^{0}$ & $57-115 \mathrm{~m}$ & Limande \\
\hline \multirow[t]{2}{*}{25} & Island-Grönl. $(2302,2305)$ & $28.3 .-4.4$ & $4,8-6,1^{0}$ & $420-480 \mathrm{~m}$ & Tiefenbarsch \\
\hline & idem $(2536,2558,2576,9579)$ & $93.8 .-5.9$ & $3,9-7,3^{0}$ & $400-480 \mathrm{~m}$ & $\begin{array}{l}\text { Tiefenbarsch } \\
\text { gesamt }\end{array}$ \\
\hline 26 & Island (2266) & 16.3. & $4-5^{0}$ & $225 \mathrm{~m}$ & gefl. Katfisch \\
\hline 27 & Grönland (2620) & 15.9 & $3-4^{0}$ & $130 \mathrm{~m}$ & Seehase \\
\hline 28 & Grönland $(2536,2568,2595)$ & $23.8 .-9.9$ & $3-6^{0}$ & $330-400 \mathrm{~m}$ & Katfisch \\
\hline 29 & Gronland $(2278,2576)$ & $19.3-5.9$ & $?-3,9^{\circ}$ & $400-480 \mathrm{~m}$ & Grenadierfisch \\
\hline 30 & Island-Grönl. $(2523,2536)$ & $21.8 .-22.8$ & $0,1-2,0^{\circ}$ & $380-400 \mathrm{~m}$ & Wolfsfisch \\
\hline 31 & Nordsee (2478) & 8.8 & $?$ & $95 \mathrm{~m}$ & Angler \\
\hline
\end{tabular}

Abkürzungen: $\mathrm{n}=$ Anzahl der Fische. Die eingeklammerte Zahl hinter $\mathrm{n}$ bedeutet Zahl der Hämoglobinbestimmungen.

Ery = Erythrozyten

Die Streuung der Einzelwerte ist in Standardabweichung angegeben. 
Ergebnisse der Blutuntersuchungen

\begin{tabular}{|c|c|c|c|c|c|c|c|c|}
\hline$=$ & 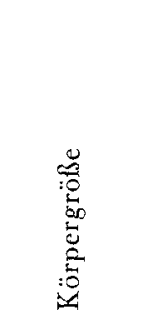 & 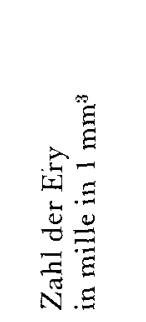 & 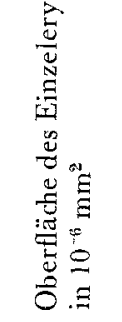 & 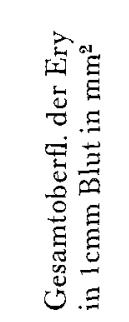 & 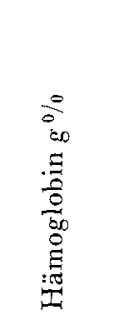 & 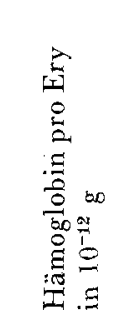 & 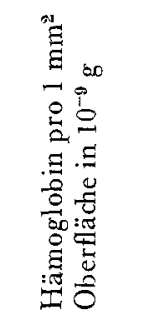 & 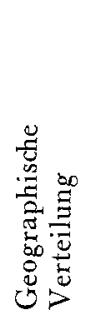 \\
\hline $8(3)$ & $30-40 \mathrm{~cm}$ & $3890 \pm 960$ & $181 \pm 28$ & $694 \pm 143$ & $14,6 \pm 1,7$ & $33,2 \pm 6,6$ & $214 \pm 0,05$ & $\mathrm{M}$ \\
\hline $3(3)$ & $15 \mathrm{~cm}$ & 3340 & 198 & 66.3 & 11,8 & $3.5,3$ & 178 & M \\
\hline $1(1)$ & $35 \mathrm{~cm}$ & 3150 & 225 & 710 & 13.5 & 42,7 & 190 & $\mathrm{MB}$ \\
\hline $16(1)$ & $19-29 \mathrm{~cm}$ & $3150 \pm 400$ & $217 \pm 17$ & $679 \pm 83$ & 16,2 & 42,5 & 262 & B \\
\hline $3(3)$ & $29-37 \mathrm{~cm}$ & 2960 & 180 & 532 & 9,2 & 31,0 & 173 & M \\
\hline $2(2)$ & $48 \mathrm{~cm}$ & 2740 & 155 & 423 & 5,1 & 20,6 & 123 & B \\
\hline $3(3)$ & $28 \mathrm{~cm}$ & 2740 & 127 & 348 & 8,1 & 30,0 & 234 & $\mathrm{M}$ \\
\hline $1(1)$ & $26 \mathrm{~cm}$ & 2690 & 152 & 409 & 9,5 & 35,3 & 232 & M \\
\hline $2(2)$ & $20-36 \mathrm{~cm}$ & 2500 & 120 & 297 & 9,1 & 44,7 & 352 & MB \\
\hline $3(3)$ & $27-29 \mathrm{~cm}$ & 2480 & 133 & 330 & 13,9 & 52.3 & 422 & M \\
\hline $1(1)$ & $37 \mathrm{~cm}$ & 2300 & 153 & 351 & 6.2 & 26.9 & 176 & BA \\
\hline $16(1)$ & $30-93 \mathrm{~cm}$ & 1860 & 201 & 369 & 5,9 & 25.1 & 177 & B \\
\hline $2(2)$ & $47-50 \mathrm{~cm}$ & 1870 & 123 & 229 & 4,6 & 24,9 & 202 & \\
\hline $2(2)$ & $45 \mathrm{~cm}$ & 1870 & 138 & 256 & 6,7 & 36,7 & 265 & \\
\hline $16(0)$ & $12-37 \mathrm{~cm}$ & $1800 \pm 250$ & $141 \pm 15$ & $253 \pm 36$ & & & & B \\
\hline $1(1)$ & $54 \mathrm{~cm}$ & 1790 & 153 & 274 & 9,9 & 51,2 & 334 & $\mathrm{BA}$ \\
\hline $15(1)$ & $31-50 \mathrm{~cm}$ & $1750 \pm 360$ & $119 \pm 26$ & $344 \pm 73$ & 7,3 & 30.5 & 242 & B \\
\hline $\mathcal{2}(0)$ & $68-70 \mathrm{~cm}$ & 1730 & 189 & 306 & & & & $\mathrm{MB}$ \\
\hline $14(0)$ & $31-50 \mathrm{~cm}$ & $1720 \pm 270$ & $168 \pm 18$ & $291 \pm 49$ & & & & B \\
\hline $1(0)$ & $62 \mathrm{~cm}$ & 1680 & 121 & 203 & & & & B \\
\hline $4(4)$ & $\begin{array}{l}44 \mathrm{~cm} \\
42 \mathrm{~cm}\end{array}$ & $1380 \pm$ & 180 & 246 & 7,4 & 53,9 & 300 & $\mathrm{BA}$ \\
\hline $6(6)$ & $32-61 \mathrm{~cm}$ & $\begin{array}{l}1840 \\
1650 \pm 410\end{array}$ & $\begin{array}{l}172 \\
175 \pm 17\end{array}$ & $\begin{array}{l}316 \\
288 \pm 75\end{array}$ & $\begin{array}{l}6,6 \\
6,9 \pm 0,7\end{array}$ & $\begin{array}{l}39,4 \\
45,8 \pm 9,1\end{array}$ & $\begin{array}{l}229 \\
260 \pm 45\end{array}$ & \\
\hline $2(2)$ & $32-38 \mathrm{~cm}$ & 1650 & 156 & 253 & 4,9 & 29,9 & 192 & $\mathrm{MB}$ \\
\hline $1(0)$ & $39 \mathrm{~cm}$ & 1630 & 175 & 286 & & & & MB \\
\hline $15(0)$ & $23-44 \mathrm{~cm}$ & 1610 & 200 & 332 & & & & MB \\
\hline $3(3)$ & $28-40 \mathrm{~cm}$ & 1410 & 161 & 227 & 4,5 & 32,3 & 200 & \\
\hline $18(3)$ & & $1580 \pm 330$ & $194 \pm 24$ & $307 \pm 78$ & & & & \\
\hline $2(0)$ & $36-43 \mathrm{~cm}$ & 1560 & 112 & 176 & & & & $\mathrm{BA}$ \\
\hline $15(0)$ & $29-45 \mathrm{~cm}$ & $1530 \pm 170$ & $187 \pm 24$ & $285 \pm 40$ & & & & B \\
\hline $3(3)$ & $27-52 \mathrm{~cm}$ & 1030 & 158 & 163 & 5,3 & 59,1 & 334 & BA \\
\hline $7(7)$ & $32-45 \mathrm{~cm}$ & 1650 & 168 & 977 & 8,7 & 53,1 & 317 & \\
\hline $10(10)$ & & $1470 \pm 410$ & $165 \pm 11$ & $242 \pm 72$ & $7,7 \pm 2,2$ & $52,8 \pm 5,2$ & $322 \pm 36$ & \\
\hline $1(1)$ & $62 \mathrm{~cm}$ & 1360 & 126 & 172 & 4,3 & 31,9 & 252 & BA \\
\hline $1(1)$ & $47 \mathrm{~cm}$ & 1340 & 167 & 223 & 5,4 & 29,7 & 244 & B \\
\hline $3(3)$ & $40-50 \mathrm{~cm}$ & 1140 & 146 & 167 & 4,5 & 39,2 & 271 & BA \\
\hline $3(3)$ & $54 \mathrm{~cm}$ & 1010 & 139 & 139 & 5,7 & 59,0 & 417 & BA \\
\hline $2(2)$ & $50 \mathrm{~cm}$ & 950 & 283 & 272 & 5,2 & 54,4 & 198 & A \\
\hline$I(1)$ & $46 \mathrm{~cm}$ & 350 & 246 & 87 & 0,9 & 25,6 & 104 & MB \\
\hline
\end{tabular}

Die Fangorte sind durch Stationsnummern bzw. die Bezeichnung N (größerer Fangbereich in der Nordsce) deutlich charakterisiert. Geographische Lage s. Abb. 1.

$\mathrm{M}=$ mediterran

$\mathrm{B}=$ boreal

$\mathrm{A}=$ arktisch 
Jahreszeitliche Unterschiede stellten sich bei Sebastes marinus und Sebastes mentella heraus.

Von einer Reihe von Arten konnten nur wenige bzw. ein Exemplar bearbeitet werden. Diese Angaben müssen angesichts der Streuungsbreite keineswegs charakteristisch für die betreffende Art sein. Doch passen sie meist gut in die Gesetzmäßigkeiten, die sich in dem besser gesicherten Material zeigen.

Faßt man die Werte aller Proben zusammen, dann besteht zwischen Erythrozytenzahl und Hämoglobingehalt des Blutes eine klare lineare Korrelation (Abbildung 2a):

$$
\begin{gathered}
\text { Korrelationskoeffizient } \mathrm{r}=0,736 \\
\mathrm{p}=0,001
\end{gathered}
$$

Dasselbe gilt für die Beziehung Gesamt-Oberfläche der roten Blutkörperchen zu Hämoglobingehalt in einer gegebenen Blutmenge (Abbildung 2b):

$$
\begin{gathered}
\text { Korrelationskoeffizient } \mathrm{r}=0,734 \\
\mathrm{p}=0,001
\end{gathered}
$$

Der Hämoglobingehalt nimmt also mit steigender Erythrozytenzahl und Gesamtoberfläche der Korpuskeln zu. In dem vorliegenden Material ist dann jedoch keine Korrelation zwischen der Zahl der Erythrozyten und der Oberfläche der einzelnen Erythrozyten erkennbar (Abbildung 2c):

\section{Korrelationskoeffizient $\mathrm{r}=0,0172$ keine statistische Sicherheit!}

Das Ergebnis der Korrelationsberechnungen wird nicht signifikant verändert, wenn nur die Durchschnittswerte pro Fischart in die Berechnung eingehen oder wenn die Daten solcher Arten, von denen nur ein oder zwei Exemplare bearbeitet wurden, aus der Berechnung ausgeschlossen werden.

Einige kleinere Versuche mit nur orientierendem Charakter wurden unternommen um festzustellen, ob das Blut frischgefangener Seefische sich von dem in Aquarien gehälterten Fischen unterscheidet. Die Fische wurden gleich nach dem Fang in Bordaquarien von der Größe $100 \times 49,3 \times 49 \mathrm{~cm}$ gesetzt und fangfrisch bzw. nach den in Tabelle 2 angegebenen Zeiten untersucht. Die Versuchsdauer konnte nur kurz gehalten werden.

Tabelle 2

Veränderungen des Blutbildes von drei Fischarten bei Hälterung im Aquarium

\begin{tabular}{cccc}
$\begin{array}{c}\text { Auf enthalt } \\
\text { im Aquarium }\end{array}$ & $\begin{array}{c}\text { Erythrozyten } \\
\text { in mille } \\
\text { in 1 cmm Blut }\end{array}$ & $\begin{array}{c}\text { Hämoglobin } \\
\mathrm{g} \%\end{array}$ & Zahl der Tiere \\
\hline Scholle & & & \\
3 Std. & 780 & 2,6 & 3 \\
24 Std. & 1960 & 5,1 & 3 \\
48 Std. & 1560 & 5,2 & \\
Bastardmakrele & & & 3 \\
0 Std. & 2470 & 13,9 & 3 \\
24 Std. & 1820 & 8,9 & \\
Streifenbrasse & & & 3 \\
O Std. & & & 3 \\
24 Std. & 2960 & 9,2 & 3
\end{tabular}


Die Schollen konnten erst nach dreistündigem Aufenthalt im Aquarium untersucht werden, wobei sich sehr niedrige Werte ergaben. Diese liegen zweifelsohne unter dem Normalmaß (vergl. Tab. 1). In den ersten drei Stunden muß also bereits eine Blutverdünnung eingetreten sein. Im Laufe des Tages steigt dann die Zahl der roten Blutkörperchen sowie der Hämoglobingehalt wieder an.

Bei der Bastardmakrele war nach 24stündigem Aufenthalt im Aquarium eine Verringerung der Erythrozytenzahl und des Hämoglobingehaltes zu beobachten.

Bei der Streifenbrasse sanken diese Werte innerhalb des ersten Tages ebenfalls, stiegen aber nach zwei Tagen wieder bis etwa unter das Initialmaß an.

Kurz nach dem Fang tritt also bei den drei untersuchten Arten eine Blutverdünnung ein. Das Blut zeigt frühestens nach einem Tag wieder Normalwerte.

\section{Diskusion}

\section{Diskussion der Methodik}

Die Schwierigkeit, Fischblut zu gewinnen und zu analysieren, wird von fast allen Bearbeitern hervorgehoben. Über die Blutentnahme wurde eingehend von Lysak (1959) und Molnar (1960) berichtet. Bei der vorliegenden Untersuchung trat eine weitere Schwierigkeit auf: die Erschwerung der Arbeit durch die Schiffsbewegungen. Aus diesem Grund mußten die bisher üblichen Verfahren auf Eignung für diesen speziellen Fall überprüft werden.

Die Herzpunktion ist an Bord häufig sehr schwer durchzuführen. Die Lage des Herzens und die Führung der Kanüle müssen genau bekannt sein. Vor allem muß sehr schnell gearbeitet werden. Daher dürfte diese Methode für Bordzwecke ausscheiden.

Ähnliches gilt für die Entnahme des Blutes durch Einführen einer Kanüle oder Kapillare in die Caudalgefäße.

Die Gewinnung des Blutes aus den Kiemen fällt schon deshalb weg, da sie bei kleinen Fischen wegen der geringen Blutmenge nicht anwendbar ist.

Das oft geübte Verfahren, den Fisch mit dem Kopf nach oben aufuhängen, den Schwanz abzuschneiden und das hervorquellende Blut aufzufangen, hat zwei große Nachteile: Es ist auf rollendem Schiff und bei der Untersuchung großer Fische unbrauchbar, da das Aufhängegerüst auf verschiedene Höhen eingestellt und der Fisch in weiteren Körperregionen befestigt werden müßten, um das Pendeln bei Seegang zu vermeiden. Der zweite Nachteil ist, daß eine sehr große Wundfläche entsteht und das Blut durch heraustretende Zellsäfte und Gewebstrümmer verunreinigt wird.

Demgegenüber dürfte die hier angewendete Methode am günstigsten sein. Das Blut läßt sich ohne umständliche Manipulationen entnehmen. Der ganz schmale, tiefe Einstich erzeugt nur eine sehr geringe Wundfläche, wodurch Verunreinigungen des Blutes auf ein Mindestmaß beschränkt werden. Diese Methode unterscheidet sich von der Blutentnahme mit der Franckschen Nadel (Dombrowski, 1953) durch die tiefere Einführung des Instrumentes. Mit dem Schnepper arbeitet man bei vielen Fischen mit dickerer Haut oder geringer Blutmenge erfolglos. 
Das Auszählen und Messen der roten Blutkörperchen gehört zu den schwierigsten Arbeiten der hämatologischen Untersuchung an Bord eines Schiffes, da mit den Schiffsbewegungen die Erythrozyten in der Zählkammer hin und herschwanken und oft die Analyse unmöglich machen. Diese Schwierigkeiten konnten mit der angewandten Photomethode mühelos beseitigt werden. Diese hat überdies noch zwei Vorteile: Erstens erfordert sie sehr wenig Zeit, so daß aus einem Fischfang hintereinander mehrere Fisch bearbeitet werden können. Sie kann auch von weniger Geübten gehandhabt werden. Zweitens ist die Genauigkeit größer, als wenn an Bord eines stark bewegten Schiffes mit dem Okularmikrometer gemessen würde. Die Photogramme können an Land ausgewertet werden. Während bei einer Sofortuntersuchung an Bord es nur bei ruhiger Schiffslage möglich ist, die nach Vorschrift und zur Erzielung einer gewissen Genauigkeit erforderlichen 80 kleinsten Quadrate der Zählkammer nach BüRKER durchzugehen, kann man nun in aller Ruhe die Zahl sämtlicher Erythrozyten feststellen, die sich in den kleinsten und größeren Quadraten und in den Rechtecken des photographierten Blickfeldes befinden. Bei einer optimalen Einstellung der Zählkammer beträgt bei der angegebenen optischen Vergrößerung die sichtbare Fläche eines Zählkammerfeldes $0,8 \mathrm{~mm}^{2} \quad(80$ kleinste Quadrate entsprechen einer Fläche von $\left.0,2 \mathrm{~mm}^{2}\right)$. Da die Auszählung der Blutkörperchen in beiden Netzfeldern der Kammer erfolgt und diese beide photographiert werden, erhält man bei guter Einstellung eine Zählfläche von $1,6 \mathrm{~mm}^{2}$. Der Zählfehler wird also wesentlich verkleinert. Ferner kann die Messung der Größe der Korpuskeln auf dem Photogramm an beliebig vielen Zellen erfolgen, wodurch wiederum eine größere Genauigkeit erzielt wird.

Die Untersuchung des Fischblutes auf fahrendem Schiff hat gegenüber der am Landaquarium durchgeführten einen Nachteil. Die Fische müssen unmittelbar nach einem Fang bearbeitet werden. Aus einem Fischzug aber können, wie in der Methodik angegeben, nur wenige, etwa vier Fische gleichzeitig von einem Mann untersucht werden. Daraus folgt, daß die Zahl der analysierbaren Exemplare eine Funktion der Zahl der Fischfänge und der Zahl der Untersucher ist.

Die Oberfläche der Erythrozyten ist angesichts der Kleinheit des Objekts sehr schwierig zu bestimmen. Ich folgte der Berechnungsweise der meisten Autoren (z. B. Schlicher, 1927; Wintrobe, 1934) und nahm als Oberfläche die doppelte Ellipsenoberfläche an. Dabei ist zwar die dritte Dimension vernachlässigt, die nur in sehr wenigen Fällen gemessen werden konnte. Jedoch stimmen die auf gleiche Weise von Bürker (1922) erhaltenen Oberflächenwerte für Säugererythrozyten mit den Werten von Drastich (1928), gewonnen durch die Hämatokrit-Methode, gut überein.

\section{Diskussion der Ergebnisse}

Bei einer vergleichenden Bearbeitung des Gefäßsystems der Vertebraten sagt Weidenneich (1933), daß bei allen Wirbeltierklassen die Größe der roten Blutkörperchen umgekehrt proportional ihrer Zahl ist. Dies wird gleichfalls von Wintrobe (1934) angenommen. Auch verschiedene Untersucher des Fischblutes (Malassez, 1872; Schlicher, 1927; Kisch, 1948; Smith et al., 1952) kommen zu dieser Schlußfolgerung. So sollen träge Fischarten sich durch eine geringe Erythrozytenzahl, aber durch eine beträchtliche Größe der Kor- 
puskeln auszeichnen, während umgekehrt aktive Schwimmer eine sehr große Zahl kleiner Erythrozyten aufweisen. Jedoch weist derselbe MALAssez schon darauf hin, daß diese Beziehung nicht immer ohne Ausnahme ist, und selbst Schlicher betont, daß die roten Blutkörperchen des Aals trotz ihrer hohen Zahl sehr groß sind. Eine umgekehrte Proportionalität zwischen Zahl und Größe wurde jedoch bisher in keinem Fall exakt nachgewiesen. Es lag daher nahe, die Berechtigung des obigen Postulats an dem eigenen Material zu überprüfen. Zur Verdeutlichung wird die Größe der roten Blutkörperchen, die sich aus Länge und Breite zusammensetzt, in einer einzigen Zahl als Oberfläche ausgedrückt. Aus der Abbildung 3 geht deutlich hervor, daß zwar eine klare Proportionalität zwischen Zahl und Gesamtoberfläche der Erythrozyten in

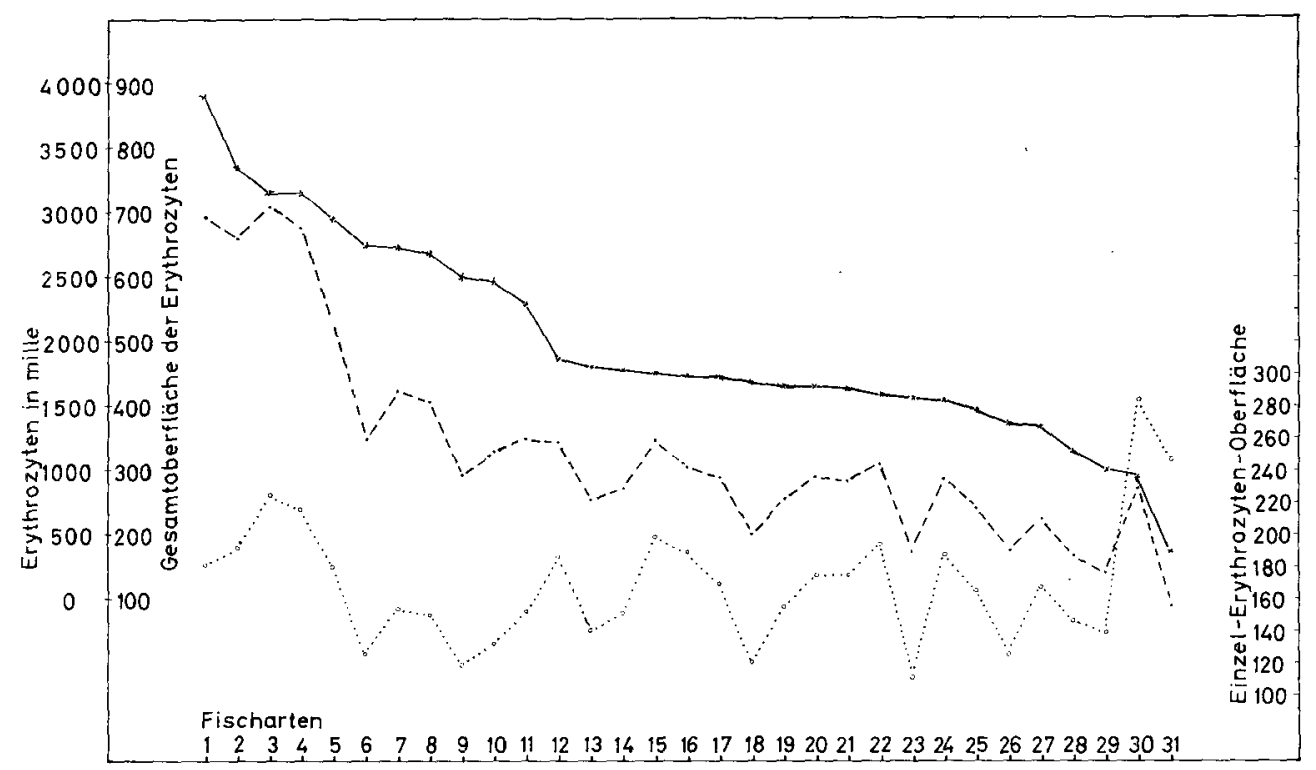

Abb. 3: Anordnung der Befunde nach steigender Erythrozytenzahl. Die Zahlen der Abszisse (Fischarten) beziehen sich auf die laufende Nummer in Tabelle 1

$$
\begin{aligned}
& \searrow \ldots \ldots \ldots \text { Erythrozytenzahl } \\
& \bigcirc \ldots \ldots \text { Oberfläche der Einzelerythrozyten } \\
& \ldots \ldots \ldots-\text { Gesamtoberfläche der Erythrozyten }
\end{aligned}
$$

einer gegebenen Blutmenge vorliegt, was auch rechnerisch bewiesen wurde (vergl. Abb. 2a, 2b), daß aber die Größe der einzelnen Erythrozyten bei den meisten Arten um einen Mittelwert schwankt, eine Korrelation der Größe mit der Zahl und der Gesamtoberfläche ist also nicht vorhanden (Abbildung 2c). So haben die Blutkörperchen der guten Schwimmer Makrele und Hering trotz ihrer großen Mengen eine mehr als mittelgroße Oberfläche. Andererseits besitzen die trägen Arten Wolfsfisch und Angler nur sehr wenig Erythrozyten, die aber bedeutend größer als die der übrigen Arten sind. Letzteres Ergebnis stimmt mit den Werten der von anderen Autoren bearbeiteten Grundfische Taugota, Opsanus tau, Lophius u. a., deren große Korpuskeln vermutlich zu obiger Annahme geführt haben, überein. Es ist aber bemerkenswert, daß einige andere, durchaus auch als "träge“ zu bezeichnende Fische wie Grenadierfisch, Seehase und Katfisch, ebenfalls nur sehr wenige Erythrozyten haben, welche indessen nur von mittlerer oder geringer Größe sind. Eine sehr kleine Zahl 
ist also nicht immer mit einer überdurchschnittlichen Größe der roten Blutkörperchen verbunden. So diskutieren mehrere Autoren(Malassez, Schlicuer, Kısch, SaIto) auch eine andere Möglichkeit, um dieses Problem zu klären, nämlich, daß die Erythrozyten um so größer sind, je niedriger ihre Träger in der Organisation bzw. Evolution oder je weiter sie abseits von der Hauptrichtung der Evolution stehen. Inwieweit dies für die einzelnen Fischarten zutrifft, läßt sich wegen unserer noch ungenügenden Kenntnis von der Evolution der Fische nicht entscheiden. Bei dem vorliegenden Material sind jedoch verwandtschaftliche Beziehungen nicht erkennbar. So sind z. B. die Streuungen der Werte innerhalb der. Pleuronectidae und der Gadidae sehr erheblich, besonders wenn man die Werte der Gesamtoberfläche vergleicht.

Aus dem Obengesagten folgt, daß der Hämoglobingehalt pro Oberflächeneinheit und der absolute Gehalt an Hämoglobin pro Erythrozyt für eine Art innerhalb einer gewissen Größenordnung liegt. Es ist jedoch festzustellen, daß die Streuung der Werte innerhalb der Species sowie von Art zu Art größer ist als bei den Säugern (Drastich, 1928).

$\mathrm{Zu}$ den das Blut charakterisierenden Merkmalen Erythrozytenzahl und Hämoglobingehalt tritt also weniger die Größe bzw. Oberfläche des einzelnen Erythrozyten, bzw. der Hämoglobingehalt pro Oberflächeneinheit oder der absolute Hämoglobingehalt des einzelnen Blutkörperchens, als vielmehr die Gesamtoberfläche der Erythrozyten, welche aber engstens mit der Zahl der Erythrozyten verknüpft ist.

Zahl und Gesamtoberfläche der Erythrozyten sowie der Hämoglobingehalt des Blutes stehen im allgemeinen in Beziehung zur Lebensweise der Fische. In diesem Zusammenhang spricht man in der Literatur von drei Gruppen von Fischen, die nach GraY (1954) folgendermaßen gekennzeichnet werden können:

1. Aktive, schnellschwimmende, in Schwärmen lebende Wanderfische, die zumindest im Sommer in ständiger Bewegung sind und sich von Plankton oder kleinen Fischen ernähren.

2. Fische von mittlerer Aktivität, die sich auf "Tageswanderungen" beschränken, sich von Crustaceen, Mollusken und sessilen Tieren ernähren und teilweise am Boden leben.

3. Sehr träge Fische, die mehr oder weniger an benthonisches Leben adaptiert sind.

So besitzen in Übereinstimmung mit den früheren Autoren die der Gruppe 1 zuzuordnenden Fische (Makrele, Finte, Hering usw.) eine hohe Zahl von Erythrozyten und einen hohen Hämoglobingehalt, die der Gruppe 2 mittlere Werte, und die Tiere der dritten Gruppe (Angler, Wolfsfisch u. a.) haben ein wenig konzentriertes Blut. Jedoch sind auch hier einige Ausnahmen zu konstatieren. So ist die Aktivität von Knurrhahn, Petermännchen und Leyerfisch wohl geringer, als es ihr recht konzentriertes Blut erwarten läßt. Dabei ist freilich zu bedenken, daß manche Arten zeitweise agil sind. Möglicherweise wurden sie bei der Untersuchung in einer Zeit erhöhter Aktivität angetroffen. Köhler und Seehecht dürften hingegen, ihrer Lebensweise entsprechend, einen höheren Erythrozyten- und Hämoglobingehalt im Blut haben. Von diesen Arten konnten leider nur 1 bzw. 2 Exemplare untersucht werden, deren Blutbild vielleicht nicht den normalen Durchschnittswert zeigt. Wintrobe (1934) fand beim Köhler 2,6 Millionen Erythrozyten und 8,1 g\% Hämoglobin. 
Vergleicht man die einzelnen Fischarten hinsichtlich ihres Fangplatzes (s. entspechende Spalte in Tab. 1) und ihres geographischen Vorkommens (s. letzte Spalte in Tab. 1), dann ist auffällig, daß eine geringere Erythrozytenzahl vorwiegend bei den borealen, boreo-arktischen und arktischen Formen anzutreffen ist. Hierzu gehören auch Arten, die zeitweise eine größere Wanderungsaktivität entfalten wie Rotbarsch und Kabeljau. Das konzentrierteste Blut hat hier der schwarze Heilbutt. Wenn auch nur eine Probe von dieser Art bearbeitet werden konnte, so paßt diese doch vorzüglich in die Theorie von der Abhängigkeit der Blutkonzentration von der Lebensweise, da dieser Fisch als einer der aktivsten Schwimmer des Nordens gilt. Der obige Befund dürfte damit zusammenhängen, daß die Fische in kälterem Wasser eine geringere Aktivität und einen geringeren Sauerstoffbedarf haben. Nach GunTER (1950) haben arktische Fische ein langsameres Wachstum und verzögerte Geschlechtsreife. Ihr Sauerstoffbedarf ist nach Scholander et al. (1953) wesentlich geringer als der von tropischen Fischen. So ist $z$. B. der nördlich von Island vorkommende Hering träger als der der Nordsee, und der Murmansk-Hering hat nach NAUmov (1956) etwa 8,5-10 $\mathrm{g} \%$ Hämoglobin, was etwa nur 2/3 der Konzentration des Herings unserer Breiten entspricht. Auch nach ScHoLANDER und VAN DAM (1957) hat das Blut arktischer Kaltwasserfische niedrige Eisenwerte, also einen niedrigen Hämoglobingehalt.

$\mathrm{Ob}$ und welchen Einfluß die Fangtiefe hat, die im Bereich Island-Grönland zwischen 200 und $480 \mathrm{~m} \mathrm{lag}$, ist noch nicht ersichtlich. VAN DAM und Scholander (1953) folgerten, daß Fische aus 390 bis $915 \mathrm{~m}$ Tiefe nicht mehr Eisen (entsprechend Hämoglobin) im Blut haben als Oberflächenfische. Jedod̀ liegt auch seinerseits nur wenig Material vor. Hier müßten weitere intensive Untersuchungen Klärung bringen.

Wie schon eingangs erwähnt wurde, haben die meisten früheren Autoren die Fische nach einer gewissen Adaptationszeit, die zum Teil nur einen Tag betrug, in einem Landaquarium untersucht. Ist das Blut solcherart gehälterter Fische von dem frischgefangener Tiere verschieden? Auch wenn das Wasser des Aquariums denselben Salz- und Sauerstoffgehalt und dieselbe Temperatur wie das Wasser am Fangort besitzt, so ist der Lebensraum doch nicht identisch: Die Druckunterschiede fehlen in den Fällen, wo die Fische in größeren Tiefen gefangen wurden, Verletzungen sind möglich. Bei längerer Hälterung können unregelmäßige Ernährung und Hungern sowie Störungen des Geschlechtszyklus (Laichzeiten) auftreten. Krankheiten sind möglich. Die Aktivität wird bei vielen Arten beeinträchtigt, besonders bei schnellschwimmenden Arten, so daß es leicht zu Sauerstoffmangel kommt wie bei der Makrele (vergl. Hall, 1930). Alle diese Faktoren können die Zusammensetzung des Blutes ändern. Aber auch bereits durch den Fang und den folgenden Transport zum Aquarium, der meist in kleinen Tragebehältern durchgeführt wird, treten häufig Störungen auf. Der Fang ruft eine Erregung des Fisches hervor, die sich bei manchen Arten durch starkes Zappeln bemerkbar macht. Dabei tritt bekanntlich Milchsäure auf, und als Folge dieser Ansäuerung entsteht Wasserentzug aus dem Blut (v. Buddenbrock, 1940), wodurch die Werte für Erythrozyten und Hämoglobin ansteigen. Nach HaLL et al. (1926) können Zustände der Atemnot bei dem agilen Brevoortia bereits nach zehnminütigem Transport in einem abgeschlossenen Behälter beobachtet werden. Nach 24 Stunden Aufenthalt im Aquarium sollen dann aber nur noch minimale Abweichungen in den Blutkomponenten festzustellen sein, also ist dann wohl der Anfangszustand 
wiederhergestellt. BAUDIN (1936) konnte ebenfalls in interessanten Versuchen am Flußbarsch zeigen, daß im Blutbild nach dem Fang starke Veränderungen auftreten, die aber erst nach vielen Tagen zu einem Ruhezustand führen. Bei seinen Untersuchungen macht sich aber zwei bis drei Stunden nach dem Fang eine Blutverdünnung bemerkbar. In den folgenden Tagen treten rhythmische Schwankungen in der Zahl der Erythrozyten und Retikulozyten und in der Sauerstoffkapazität auf, bis am fünften bis achten Tag das Blutbild zunächst wieder normal wird. Aber auch dann erfolgt eine weitere Veränderung in Form einer Anämie.

Wie aber verhalten sich andere Fischarten?

Aus den eigenen Versuchen geht hervor, daß während der Hälterung im Bordaquarium ebenfalls Unregelmäßigkeiten im Blutbild zu bemerken sind. Bei allen drei Arten tritt zunächst eine Hypoglobulie auf. Die Ergebnisse von BAUDIN werden damit auch bei anderen Arten bestätigt.

Es ist wohl anzunehmen, daß ein ähnliches Verhalten noch bei weiteren Arten zu finden ist. Daraus ist zu schließen, daß genaue Werte nur dann gewonnen werden können, wenn die Fische sofort nach dem Fang am Fangort untersucht werden. Ist dies nicht möglich, dann muß man eine sehr lange Zeit adaptieren lassen. Jedoch sind die dann erhaltenen Werte auch noch fraglich, weil die andersartigen Lebensbedingungen weitere Veränderungen des Blutbildes hervorrufen können.

Ein Vergleich der eigenen Beobachtungen mit den Ergebnissen anderer Autoren zeigt, wenn man die großen bei den Fischen vorkommenden Schwankungen der Blutwerte berücksichtigt, größenordnungsmäßig Übereinstimmung, doch sind auch einige größere Abweichungen wahrzunehmen. Es ist nicht zu entscheiden, ob diese Differenzen durch große individuelle Streuungen, methodische Fehler oder durch Aquariumsverhältnisse bedingt sind. Abweichungen in der Größe der Erythrozyten können dadurch entstehen, daß bei den eigenen Untersuchungen die Blutkörperchen in Hayemscher Lösung gemessen wurden, welche bekanntlich keine Volumenveränderung bewirkt. Im angelsächsischen Raum erfolgt jedoch die Messung vielfach erst im fixierten Präparat, wodurch die Erythrozyten zusammenschrumpfen. Es ist durchaus denkbar, daß hierbei die Erythrozyten verschiedener Arten in unterschiedlichem Maße Größenveränderungen erleiden, so daß die Angaben mehrerer Autoren bei Anwendung verschiedener Meßmethoden überhaupt nicht mehr vergleichbar sind.

\section{Zusammenfassung}

Es wird eine Methode beschrieben, die es gestattet, den Erythrozytenund Hämoglobingehalt im Blut von Seefischen an Bord eines fahrenden Schiffes zu bestimmen. Bei starken Schiffsbewegungen werden die ErythrozytenZählkammern photographiert. Die Photogramme können an Land ausgewertet werden.

31 Seefischarten wurden direkt am Fangort in der Nordsee und im Nordatlantik unmittelbar nach dem Fang untersucht. Erythrozytenzahl und -größe und Hämoglobingehalt wurden gemessen. Daraus konnte die Gesamtoberfläche der Erythrozyten und der Hämoglobingehalt pro Blutkörperchen und pro Oberflächeneinheit berechnet werden.

Das Blutbild einer großen Zahl noch nicht bearbeiteter Arten wurde beschrieben. 
Die Fangorte sind durch Position, Datum, Angaben über Tiefe, Temperatur, Sauerstoff- und Salzgehalt charakterisiert.

Erythrozytenzahl, Gesamtoberfläche der Erythrozyten und Hämoglobingehalt zeigen deutliche Beziehungen zur Aktivität und zum geographischen Vorkommen der Fische.

Zwischen Erythrozytenzahl und Hämoglobingehalt des Fischblutes besteht eine signifikante lineare Korrelation. Dasselbe gilt für die Beziehung zwischen Gesamtoberfläche der Erythrozyten und dem Hämoglobingehalt.

Zwischen der Zahl der Erythrozyten und ihrer Größe konnte keine Korrelation gefunden werden. Nicht alle trägen Fische haben große rote Blutkörperchen.

Orientierende Versuche mit drei Fischarten deuten an, daß für längere Zeit im Blutbild Veränderungen auftreten, wenn man Seefische nach dem Fang in ein Aquarium versetzt. Schon einige Stunden nach dem Fang ist eine Blutverdünnung zu beobachten.

Die Arbeit wurde in der Fischereibiologischen Abteilung des Instituts für Meereskunde der Universität Kiel begonnen und in der Biologischen Anstalt Helgoland beendet. Herrn Prof. Dr. Kändler, der die Untersuchungen anregte, Herrn Prof. Dr. Bückmann, Hamburg, und Herrn Dr. Aurich, List auf Sylt, danke ich herzlich für ihre Gastfreundschaft und vielfache Unterstützung. Mein Dank gilt ferner Herrn cand. Tekin Mengi, Kiel, welcher einen Teil der Analysen an den Nordseefischen durchführte, Herrn Dr. Ziegelmeier, List, für die Unterstützung bei der Ausarbeitung der Photomethode und Herrn Prof. Dr. Kalle, Hamburg, für die Zurverfügungstellung der hydrographischen Daten.

\section{Literaturverzeichnis}

Baudin, L., 1936: Modifications du sang du poisson Perca fluviatilis consécutives à la pêche en profondeur. C. R. Soc. Biol. 121, 437.

B e r g, L. S, System der rezenten und fossilen Fischartigen und Fische. Deutscher Verlag der Wissenschaften, Berlin 1958.

Betke, K., und Savelsberg, W., 1949/50: Stufenphotometrische Hämoglobinbestimmung mittels Cyanhämiglobin. Biochem. Z. 320, 431.

v. Buddenbrock, W., 1940: Die physiologischen Arbeiten für die Deutsche Wissenschaftliche Kommission für Meeresforschung. Ber. Dt. wiss. Komm. Meersforsch. 9, 218.

Bürker, K., 1922: Das Gesetz der Verteilung des Hämoglobins auf die Oberfläche der Erythrozyten. Pflügers Arch. 195, 516.

van Dam, L., and Scholander, P. F., 1953: Concentration of hemoglobin in the blood od deep-sea fishes. J. cell. comp. Physiol. 41, 522.

Dombrowski, H., 1953: Untersuchungen über das Blut des Karpfens (Cyprinus carpio L.) und einiger anderer Süßwasserfischarten. Biol. Zb1. 72, 182.

Drastich, L., 1928: Ist die Konzentration des Blutfarbstoffs im Blutkörperchen bei allen Tieren konstant? Pflügers Arch. 219, 227.

Druckrey. H., Danneberg, P., Kaiser, K., Fromme, L., und Schneider, H., 1952: Zur Methodik der Hämoglobin-Bestimmung. Biochem. Z. 322, 535.

Gray, I. E., 1954: Comparative study of the gill area of marine fishes. Biol. Bull. 107, 219.

Gunter, G., 1950: Correlation between temperature of water and size of marine fishes on the Atlantic and Gulf coasts of the United States. Copeia, No. 4, 298.

Hall, F. G., 1930: The ability of the common mackerel and certain other marine fishes to remove dissolved oxygen from sea water. Amer. J. Physiol. 93, 417.

Hall, F. G. and Gray, I. G., 1929: The hemoglobin concentration of the blood of marine fishes. J. Biol. Chem. 81, 589.

Hall, F. G., Gray, I. G., and Lepkovsky, S., 1926: The influence of asphyxiation on the blood constituents of marine fishes. J. Biol. Chem. 67, 549

Kisch, B., 1948: Studies on the red blood cells of fish. Biol. Bull. 95, 266.

Lysak, A., 1959: Die Blutentnahme von Fischen zu diagnostischen Zwecken sowie deren Einfluß auf das spätere Blutbild und den Zuwachs. Acta Hydrobiol. 1, 37. 
Malassez, M. L., 1872: De la numération des globules rouges du sang chez les mammifères, les oiseaux et les poissons. C. R. Acad. Sci. 75, 1528.

Molnár, G., 1960: Methode der Blutentnahme für hämatologische Untersuchungen bei Fischen. Z. f. Fisch. Hilfswiss. 9, N. F., 101.

Naumov, W. M., 1956: Ovogenese und Okologie des Geschlechtscyclus beim MurmanskHering. Knipowitsch Institut Murmansk, IX.

Norman, J. R., 1934: A systematic monograph of the flatfishes (Heterosomata). London, Oxford University Press.

Saito. K., 1954: Biochemical studies on the fish blood. I. On the morphological property of blood-corpusceles. Bull. Jap. Soc. Sci. Fish., 19, 1134.

Schlicher, J., 1927: Vergleichend-physiologische Untersuchungen der Blutkörperchen-Zahlen bei Knochenfischen. Zool. Jahrb. Allg. Zool. 43, 121.

Scholander, P. F., Flagg, W., Walters, V.. and Irving, L., 1953: Climatic adaptation in arctic and tropical poikilotherms. Physiol. Zool. 26, 67.

Scholander, P. F., and van Dam, L., 1957: The concentration of hemoglobin in some coldwater arctic fishes. J. cell. comp. Physiol. 49, 1.

Smith, Ch. G., Lewis, W. M., and Kaplan, H. M., 1952: A comparative morphologic and physiologic study of fish blood. The progressive Fish-Culturist 14, 169.

Weidenreich, F., 1933: Gefäßsystem. in: Handbuch der vergleichenden Anatomic der Wirbeltiere. 6. Bd. Urban \& Schwarzenberg, Berlin-Wien.

Wintrobe, M. M., 1934: Variations in the size and hemoglobin content of erythrocytes in the blood of various vertrebrates. Folia haematologica 51, 32. 